$\begin{array}{ll}\text { Research Square } & \begin{array}{l}\text { Preprints are preliminary reports that have not undergone peer review. } \\ \text { They should not be considered conclusive, used to inform clinical practice, } \\ \text { or referenced by the media as validated information. }\end{array}\end{array}$

\title{
Association Between Social Support and Mental Health in Pre-Old and Old Age: Is It Different Depends on the Object? A Cross-Sectional Study
}

\author{
Yaya Li \\ Osaka University Graduate School of Medicine Division of Health Sciences \\ Mai Kabayama ( $\square$ kabayama@sahs.med.osaka-u.ac.jp) \\ Osaka Shiritsu Daigaku https://orcid.org/0000-0003-1394-1152 \\ Kei Kamide \\ Osaka University Graduate School Division of Health Sciences
}

Research article

Keywords: Social support, Mental health, Community dwellers, Pre-old age, Old age, Objects, Japan

Posted Date: June 30th, 2020

DOI: https://doi.org/10.21203/rs.3.rs-36076/v1

License: (c) (i) This work is licensed under a Creative Commons Attribution 4.0 International License. Read Full License 


\section{Abstract}

Background The association of social support with mental health for the older adults has been well documented; however, object-specific support was rarely discussed. This study examined the relationships of multiple aspects of social support, especially focusing on objects of social support, across different aging stages.

Methods We conducted a self-reported cross-sectional survey in Osaka, Japan in 2013 . We analyzed 659 pre-old and 888 old age participants. SF-36v2 was used to measure mental health. Objects of social support was divided into 3 categories (kin, friends, community) with integrating sub-dimensions of social support such as instrumental / emotional social support (function) and provided / received social support (role). Ordinary least squares, controlled for covariates, was carried out to analyze the variation among three objects of social support on mental health in two age groups.

Results The results showed a positive association within the pre-old age group but not within the old age group. For the pre-old age group, either emotional or instrumental social support either provided to or received from a community neighbor had a positive association, while emotional social support provided to kin or friends and instrumental support received from friends didn't show any relation to mental health.

Conclusions The relationship between social support and mental health varied by objects and age groups.

\section{Background}

Successful mental health aging has become a topic worldwide, especially in aging countries. Fifteen percent of adults may suffer a mental disorder [1]. Social support, as a positive type of social network [2], has a beneficial association with mental health in the older people. Social support has a positive link with happiness [3], depression [4, 5], life stress [6], reduced social isolation [7], and lower death risk or higher survival $[8,9]$. In addition, there is a moderate relationship between mental status and risk factors [10, 11]. Making clear which kind of social support is more beneficial has become an important topic in epidemiology and a priority for community-based invention policies.

The association between mental health and social support networks is more complicated when it comes to the aging progress. Graham and Pozuelo [12] illustrated a U-shaped curve relationship between age and mental health, with older people tending to be happier. On the other hand, changes of the personal network were common issues along with illness, or decreasing functional ability with aging [13]; for example, more connection with relatives [14], a reduction in social network [15], a decline in social support [16] or less help provided [17].

Social support is commonly subdivided into informal and formal support [18], perceived and received support [8, 11], and emotional and instrumental support [18-20]. However, previous reports overlooked the object-specific pattern. Previous studies attempted to show the effect of resources of social support [21-23]; however, the discussions were limited to: a) close relationships such as kin or friends, even though community already plays a role in health policies; b) a recipient view in a single-track supportive relationship that neglected samples as providers of social support, although discussion on reciprocity of both giving and taking also matter [24, 25].

Therefore, a dynamic view of mental health and social support especially focused on the differences among objects of social support in different aging stages is needed.

The present study aimed to investigate the relationship between social support and mental status based on the objects of social support in different aging stages by integrating sub-dimensions of support such as functions (instrumental / emotional) and roles (provider / receiver).

\section{Methods}

\subsection{Design and participants}

A cross-sectional community-based mail survey was conducted in the city of Hirakata, Osaka, Japan in 2013. Hirakata City has a population of 0.41 million of which approximately $23.4 \%$ of people were aged over 65 in Oct. 2013 [26].

General residents aged 65 to 89 years who were living in a Hirakata-city were randomly picked from the residential registration using quota sampling stratified by age, sex, and area. In total, 3600 community dwellers were mailed an anonymous questionnaire from Dec. 2013 to May 2014.

The protocol of the study was approved by the ethics committee at the Division of Health Sciences, Osaka University Graduate School of Medicine (approval number 271).

\subsection{Measures}




\subsubsection{Social support}

Objects of social support; the present study measured social support depending on different objects in daily supportive interaction. Given the lack of previous studies that operationalized the norms of social support by objects, proxemics about interpersonal distance [27] were used to divide the objects into three categories: intimate objects (family or relatives), personal objects (friends), social objects (community neighbors).

The sub-dimensions of social support: 1) function (emotional social support / instrumental social support), 2) role (provider / receiver of social support). 1)Function; considering the widely accepted definition of social support as "the emotionally or instrumentally sustaining quality of social relationships" [28], emotional social support (ESS) and instrumental social support (ISS) were used as the functional facets of social support [29]. ESS was measured by two items: caring for people and listening to people's feelings or troubles. ISS was evaluated by three items: taking care of people who got sick with mild or heavy symptoms and caretaking. 2)Role; all five specific questions were asked two times to separately assess situation-specific outcomes as the participants have the role of a provider or a receiver in an interpersonal connection. All items were binary variables with 1 representing having social support and 0 representing no social support. Each participant who answered positively for any of the items of each sub-dimension were viewed as having social support.

Participants were asked whether the ESS or ISS they received came from family/relatives, friends or community neighbors; or whether they gave their ESS or ISS to family/relatives, friends or community neighbors.

\subsubsection{Mental health}

A Mental Component Summary (MCS), was collected by SF-36 (MOS 36-Item Short-Form) Japanese version 1.2 to quantify mental health. SF36 is a widely used scale in more than 170 countries for health outcomes [30]. The Japanese version has been proved with high validity and reliability [31, 32].

In the present study, MCS was adopted as a part of a three-component model (MCS, PCS, and RCS) which is suggested to be used in Japan [33]. The score was a 0-100 score calculated by five subscales: General Health (five items), Role Emotional (five items), Bodily Pain (two items), Vitality (four items), and Mental Health (five items). Each relevant item was a 5- or 6-point scale. A higher MCS score meant better mental health status.

All the scoring, coping with missing values, and calculation of MCS were conducted following the published guidelines [34].

\subsubsection{Age Groups}

The two age groups were defined as pre-old age (65 to 74 years old) group and old age (75 to 89 years old) group, using the definition by the Joint Committee of Japan Gerontological Society and the Japan Geriatrics Society [35], which has been accepted by the government of Japan as the standard.

\subsubsection{Independent variables}

Demographic variables included gender, equivalent income, present chronic illness, and physical function as covariates [36]. The variables of having child/children and living arrangement were also taken into account to control for potential support.

\subsection{Statistical analysis}

Descriptive statistics were conducted to show the basic characteristics. Then, the demographics and features of social support were compared between the two age groups by chi-square test. Multiple liner regression was carried out to assess the relationship between mental health and objects of specific sub-dimensions of social support (received ESS, provided ESS, received ISS, provided ISS) by enrolling each of the three object categories (kin, friends, community neighbors) respectively. All regression models were adjusted by gender, equivalent income, present illness, long-term care need, living status and having children. All the data were conducted with SPSS v24.0 with a level of significance at 0.05.

\section{Results}

\subsection{Demographic characteristics}

The response rate was $43.0 \%(n=1547)$. Table 1 shows the demographic characteristics between the two age groups.

We analyzed 659 pre-old age individuals and 888 old age participants. Overall, the old age group had better mental health outcomes than the pre-old age group ( $p=0.007)$; however, both groups showed similar numbers to the national standard (52.3 \pm 9.2 for people in their $60 \mathrm{~s}$ and $53.5 \pm 9.5$ people in their $70 \mathrm{~s}$ in 2007) [34]. The analytic sample contained more males, especially in the old age group ( $p=0.006)$. Most of the 
participants had chronic illness while the old age group had a higher proportion $(p=0.000)$; however, most respondents did not require longterm care, especially in the pre-old groups $(p=0.000)$.

Table 1

Demographic and social economic characteristics between two age groups

\begin{tabular}{|c|c|c|c|}
\hline Characteristic & Pre-old ( $n=659)$ & Old $(n=888)$ & p value ${ }^{a}$ \\
\hline Mental health, meas \pm SD $b$ & $52.8 \pm 9.2$ & $54.2 \pm 9.2$ & 0.007 \\
\hline Sex, $n(\%)$ & & & 0.006 \\
\hline male & $344(52.2)$ & $526(59.2)$ & \\
\hline female & $315(47.8)$ & $362(40.8)$ & \\
\hline Equivalent income, $\mathrm{n}(\%)$ & & & 0.271 \\
\hline$<100$ & $49(8.2)$ & $77(10.0)$ & \\
\hline $100-199$ & $253(42.4)$ & $345(44.9)$ & \\
\hline $200-299$ & $188(31.5)$ & $212(27.6)$ & \\
\hline $300-399$ & $35(5.9)$ & $56(7.3)$ & \\
\hline $400-499$ & $49(8.2)$ & $48(6.2)$ & \\
\hline$>=500$ & $22(3.7)$ & $31(4.0)$ & \\
\hline Present illness, n(\%) & & & 0.000 \\
\hline No recent illness & 139(21.9) & $87(10.4)$ & \\
\hline Having present illness & 497(78.1) & $751(89.6)$ & \\
\hline Physical function, n(\%) & & & 0.000 \\
\hline Not need long-term care & $642(98.6)$ & $773(89.6)$ & \\
\hline Need long-term care & $9(1.4)$ & $90(10.4)$ & \\
\hline Dwelling condition, n(\%) & & & 0.000 \\
\hline Living alone & $74(11.4)$ & $152(17.9)$ & \\
\hline Not living alone & $576(88.6)$ & $695(82.1)$ & \\
\hline Children, n(\%) & & & 0.984 \\
\hline No child/children & $53(8.3)$ & $70(8.3)$ & \\
\hline Having child/children & $589(91.7)$ & 775(91.7) & \\
\hline \multicolumn{4}{|c|}{ a P-value is the comparison between pre-old groups and old age groups using Chi-square test or T-test (mental health); } \\
\hline
\end{tabular}

Most people were not living alone; however, there were more people living alone in the old age group $(p=0.000)$, even though most of them had children. The two age groups' equivalent incomes showed they weren't disadvantaged and were consistent with national data (medium of 244) [37].

\subsection{Social support characteristics}

Table 2 shows the social support based on the objects between the two age groups. Social support is divided by the sub-dimensions (functions and roles). 
Table 2

Sub-dimensions of social support between the two age groups

\begin{tabular}{|c|c|c|c|c|c|c|}
\hline \multicolumn{4}{|l|}{ Characteristic, n(\%) } & \multirow{2}{*}{$\begin{array}{l}\text { Pre-old } \\
(n=659)\end{array}$} & \multirow{2}{*}{$\begin{array}{l}\text { Old } \\
(n=888)\end{array}$} & \multirow[t]{2}{*}{ p value ${ }^{a}$} \\
\hline$S S^{b}$ & Functions & Roles & Objects & & & \\
\hline \multirow[t]{12}{*}{ Having social support } & \multirow[t]{6}{*}{ Emotional social support } & \multirow[t]{3}{*}{ Receiver } & Family or relatives & $542(87.4)$ & $660(84.8)$ & 0.166 \\
\hline & & & Individual friends & $358(57.7)$ & $323(41.5)$ & 0.000 \\
\hline & & & Community neighbors & $148(23.9)$ & $187(24.0)$ & 0.943 \\
\hline & & \multirow[t]{3}{*}{ Provider } & Family or relatives & $521(85.0)$ & $566(77.5)$ & 0.001 \\
\hline & & & Individual friends & $336(54.8)$ & $273(37.4)$ & 0.000 \\
\hline & & & Community neighbors & $159(25.9)$ & $182(24.9)$ & 0.673 \\
\hline & \multirow[t]{6}{*}{ Instrumental social support } & \multirow[t]{3}{*}{ Receiver } & Family or relatives & $569(92.4)$ & $696(90.5)$ & 0.221 \\
\hline & & & Individual friends & $116(18.8)$ & $96(12.5)$ & 0.001 \\
\hline & & & Community neighbors & 115(18.7) & $129(16.8)$ & 0.358 \\
\hline & & \multirow[t]{3}{*}{ Provider } & Family or relatives & $522(86.4)$ & $529(76.7)$ & 0.000 \\
\hline & & & Individual friends & $144(23.8)$ & 101(14.6) & 0.000 \\
\hline & & & Community neighbors & $120(19.9)$ & $119(17.2)$ & 0.225 \\
\hline \multicolumn{4}{|l|}{ No social support } & 11(1.8) & 18(2.3) & 0.502 \\
\hline \multicolumn{7}{|c|}{ 4. a P-value is the comparison between pre-old groups and old age groups using Chi-square test; } \\
\hline
\end{tabular}

Most participants were either a provider or a receiver. About $2 \%$ of respondents were not in a social supportive interaction. Emotional social support was provided by or to family or relatives (more than $80 \%$ ) and individual friends (more than $50 \%$ ) rather than community neighbors (approximately 25\%). When we investigated instrumental social support, family or relatives became the major object (almost $90 \%$ in a provided social support context; about $80 \%$ in a received social support context).

As shown in Table 2, differences between the two age groups were found on all the sub-dimensions of social support with individual friends. Compared to the old age group, pre-old age people were more connected with friends when it comes to ESS or ISS (received ESS: $p=0.000$; provided ESS: $p=0.000$; received ISS: $p=0.001$; provided ISS: $p=0.000$ ) and provided more emotional or instrumental social support to their family or relatives (ESS: $p=0.001$; ISS: $p=0.000$ ). No significant differences were found on social support with community neighbors.

\subsection{Relations between mental health and social support based the objects in two age stages}

Table 3 to Table 6 (see Additional file 1,2,3,4) show the results of multiple regression models, which represent the associations between mental health and social support (ESS and ISS) based on the objects in the pre-old and the old age groups stratified by the roles (receiver / provider). Each table contains 6 models; Models 1 to 3 illustrate results in the pre-old age group while Models 4 to 6 show the results in the old group. Models 1 and 4, Models 2 and 5, Models 3 and 6 depict the results of social support with kin, friends, and community, respectively. Each model in each table was adjusted by gender, equivalent income, present illness, physical function, dwelling condition, children.

Table 3 (see Additional file 1) shows the relationship between received ESS and mental health based on the age groups. ESS from family or relatives, friends, and community neighbors had a positive association with mental health status in the pre-old age group (Models 1 to 3 ). Support from community neighbors $(\beta=0.146, p=0.001)$ was stronger than individual friends $(\beta=0.126, p=0.007)$ and kin $(\beta=0.090, p=$ 0.048), also explained the most variation (R2 $=0.052)$. In terms of provided ESS (Table 4; see Additional file 2 ), only support to community neighbors yielded a positive coefficient $(\beta=0.112, p=0.015)$. Variance among different objects in received or provided ESS were not found in the old age group (Models 4, 5 and 6 in Table 3 and Table 4).

Table 5 (see Additional file 3 ) shows the results of received ISS. The pre-old age people who were receiving ISS from family or relatives $(\beta=$ $0.202, p=0.000)$ and community neighbors $(\beta=0.147, p=0.001)$ had better mental health outcomes; received ISS from kin explained more 
variation $(\mathrm{R} 2 \mathrm{=}$ 0.068). For the old age group (Models 4, 5, 6), ISS with different objects showed a positive association; however, this was not statistically significant.

In terms of provided ISS (Table 6; see Additional file 4), we found positive association with mental health among all of the three types of objects in the pre-old group (Models $1,2,3$ ). The relative higher numbers were the results of support provided to family or relatives $(\beta=0.152, p$ $=0.001)$ and individual friends $(\beta=0.145, p=0.001)$. The variable of giving social support to community dwellers also showed a significant relationship $(\beta=0.115, p=0.011)$, even with a lower variance explanation ( $2=0.044)$. A significant coefficient for objects in the old age group wasn't found (Models 4, 5, 6).

Tables 3-6 show a negative relationship between chronic illness and mental health in both age groups. For the old age group, long-term care status showed similar results in all four tables.

Table 3. Multiple liner regression analysis modeling for objects of received emotional social support

\begin{tabular}{|c|c|c|c|c|c|c|c|c|c|c|c|c|c|}
\hline & & \multicolumn{6}{|c|}{ Pre-old (n = 659) } & \multicolumn{6}{|c|}{ Old $(n=888)$} \\
\hline & & \multicolumn{2}{|c|}{ Model 1} & \multicolumn{2}{|c|}{ Model 2} & \multicolumn{2}{|c|}{ Model 3} & \multicolumn{2}{|c|}{ Model 4} & \multicolumn{2}{|c|}{ Model 5} & \multicolumn{2}{|c|}{ Model 6} \\
\hline & & $\beta^{a}$ & $\begin{array}{l}\mathrm{p} \\
\text { value }\end{array}$ & $\beta^{a}$ & $\begin{array}{l}\mathrm{p} \\
\text { value }\end{array}$ & $\beta^{\mathrm{a}}$ & $\begin{array}{l}\mathrm{p} \\
\text { value }\end{array}$ & $\beta^{\mathrm{a}}$ & $\begin{array}{l}\mathrm{p} \\
\text { value }\end{array}$ & $\beta^{\mathrm{a}}$ & $\begin{array}{l}\mathrm{p} \\
\text { value }\end{array}$ & $\beta^{\mathrm{a}}$ & $\begin{array}{l}\mathrm{p} \\
\text { value }\end{array}$ \\
\hline \multirow{6}{*}{$\begin{array}{l}\text { Adjusted } \\
\text { variables }\end{array}$} & Female & -0.048 & 0.276 & -0.080 & 0.084 & -0.070 & 0.119 & 0.043 & 0.336 & 0.040 & 0.374 & 0.048 & 0.284 \\
\hline & $\begin{array}{l}\text { Equivalent } \\
\text { income }\end{array}$ & 0.105 & 0.018 & 0.089 & 0.047 & 0.110 & 0.013 & 0.070 & 0.116 & 0.065 & 0.144 & 0.074 & 0.094 \\
\hline & $\begin{array}{l}\text { Having } \\
\text { present } \\
\text { illness }\end{array}$ & -0.165 & 0.000 & -0.165 & 0.000 & -0.166 & 0.000 & -0.113 & 0.010 & -0.114 & 0.009 & -0.109 & 0.013 \\
\hline & $\begin{array}{l}\text { Need long- } \\
\text { term care }\end{array}$ & 0.008 & 0.861 & 0.017 & 0.710 & -0.006 & 0.901 & -0.127 & 0.004 & -0.123 & 0.006 & -0.127 & 0.004 \\
\hline & $\begin{array}{l}\text { Not living } \\
\text { alone }\end{array}$ & -0.041 & 0.376 & -0.010 & 0.818 & -0.016 & 0.725 & -0.020 & 0.652 & -0.019 & 0.678 & -0.029 & 0.521 \\
\hline & $\begin{array}{l}\text { Having } \\
\text { child/children }\end{array}$ & 0.058 & 0.208 & 0.062 & 0.176 & 0.059 & 0.198 & 0.068 & 0.121 & 0.067 & 0.131 & 0.067 & 0.129 \\
\hline \multirow[t]{6}{*}{ Objects } & $\begin{array}{l}\text { Family or } \\
\text { relatives }\end{array}$ & 0.090 & 0.048 & & & & & 0.004 & 0.927 & & & & \\
\hline & $\begin{array}{l}\text { Individual } \\
\text { friends }\end{array}$ & & & 0.126 & 0.007 & & & & & 0.030 & 0.495 & & \\
\hline & $\begin{array}{l}\text { Community } \\
\text { dwellers }\end{array}$ & & & & & 0.146 & 0.001 & & & & & -0.059 & 0.185 \\
\hline & $\mathrm{R} 2^{\mathrm{b}}$ & 0.039 & & 0.045 & & 0.052 & & 0.03 & & 0.031 & & 0.033 & \\
\hline & $\mathrm{N}$ & 497 & & 497 & & 497 & & 520 & & 520 & & 520 & \\
\hline & $\mathrm{F}$ & 3.88 & & 4.372 & & 4.901 & & 3.281 & & 3.349 & & 3.542 & \\
\hline
\end{tabular}

a $\beta$, standardized coefficients;

b R2, adjusted coefficient of determination; 
Table 4. Multiple liner regression analysis modeling objects of provided emotional social support

\begin{tabular}{|c|c|c|c|c|c|c|c|c|c|c|c|c|c|}
\hline & & \multicolumn{6}{|c|}{ Pre-old $(n=659)$} & \multicolumn{6}{|c|}{ Old $(n=888)$} \\
\hline & & \multicolumn{2}{|c|}{ Model 1} & \multicolumn{2}{|c|}{ Model 2} & \multicolumn{2}{|c|}{ Model 3} & \multicolumn{2}{|c|}{ Model 4} & \multicolumn{2}{|c|}{ Model 5} & \multicolumn{2}{|c|}{ Model 6} \\
\hline & & $\beta^{a}$ & $\begin{array}{l}\mathrm{p} \\
\text { value }\end{array}$ & $\beta^{a}$ & $\begin{array}{l}\mathrm{p} \\
\text { value }\end{array}$ & $\beta^{a}$ & $\begin{array}{l}\mathrm{p} \\
\text { value }\end{array}$ & $\beta^{a}$ & $\begin{array}{l}\mathrm{p} \\
\text { value }\end{array}$ & $\beta^{a}$ & $\begin{array}{l}\mathrm{p} \\
\text { value }\end{array}$ & $\beta^{a}$ & $\begin{array}{l}\mathrm{p} \\
\text { value }\end{array}$ \\
\hline \multirow{6}{*}{$\begin{array}{l}\text { Adjusted } \\
\text { variables }\end{array}$} & Female & -0.050 & 0.263 & -0.063 & 0.165 & -0.075 & 0.103 & 0.033 & 0.480 & 0.026 & 0.581 & 0.033 & 0.476 \\
\hline & $\begin{array}{l}\text { Equivalent } \\
\text { income }\end{array}$ & 0.099 & 0.029 & 0.100 & 0.025 & 0.112 & 0.012 & 0.074 & 0.103 & 0.070 & 0.123 & 0.074 & 0.105 \\
\hline & $\begin{array}{l}\text { Having } \\
\text { present } \\
\text { illness }\end{array}$ & -0.165 & 0.000 & -0.166 & 0.000 & -0.162 & 0.000 & -0.110 & 0.014 & -0.112 & 0.012 & -0.110 & 0.014 \\
\hline & $\begin{array}{l}\text { Need long- } \\
\text { term care }\end{array}$ & 0.005 & 0.903 & 0.008 & 0.853 & 0.003 & 0.940 & -0.132 & 0.003 & -0.125 & 0.006 & -0.131 & 0.003 \\
\hline & $\begin{array}{l}\text { Not living } \\
\text { alone }\end{array}$ & -0.028 & 0.538 & -0.019 & 0.684 & -0.020 & 0.669 & -0.016 & 0.736 & -0.016 & 0.724 & -0.017 & 0.711 \\
\hline & $\begin{array}{l}\text { Having } \\
\text { child/children }\end{array}$ & 0.062 & 0.174 & 0.062 & 0.177 & 0.056 & 0.218 & 0.051 & 0.257 & 0.048 & 0.286 & 0.051 & 0.259 \\
\hline \multirow[t]{6}{*}{ Objects } & $\begin{array}{l}\text { Family or } \\
\text { relatives }\end{array}$ & 0.060 & 0.183 & & & & & -0.008 & 0.854 & & & & \\
\hline & $\begin{array}{l}\text { Individual } \\
\text { friends }\end{array}$ & & & 0.087 & 0.055 & & & & & 0.041 & 0.375 & & \\
\hline & $\begin{array}{l}\text { Community } \\
\text { dwellers }\end{array}$ & & & & & 0.112 & 0.015 & & & & & 0.001 & 0.983 \\
\hline & $\mathrm{R} 2^{\mathrm{b}}$ & 0.035 & & 0.038 & & 0.043 & & 0.027 & & 0.029 & & 0.027 & \\
\hline & $\mathrm{N}$ & 494 & & 494 & & 494 & & 499 & & 499 & & 499 & \\
\hline & $\mathrm{F}$ & 3.535 & & 3.824 & & 4.161 & & 3.013 & & 3.125 & & 3.008 & \\
\hline
\end{tabular}


Table 5. Multiple liner regression analysis modeling objects of received instrumental social support

\begin{tabular}{|c|c|c|c|c|c|c|c|c|c|c|c|c|c|}
\hline & & \multicolumn{6}{|c|}{ Pre-old $(n=659)$} & \multicolumn{6}{|c|}{ Old $(n=888)$} \\
\hline & & \multicolumn{2}{|c|}{ Model 1} & \multicolumn{2}{|c|}{ Model 2} & \multicolumn{2}{|c|}{ Model 3} & \multicolumn{2}{|c|}{ Model 4} & \multicolumn{2}{|c|}{ Model 5} & \multicolumn{2}{|c|}{ Model 6} \\
\hline & & $\beta^{\mathrm{a}}$ & $\begin{array}{l}p \\
\text { value }\end{array}$ & $\beta^{\mathrm{a}}$ & $\begin{array}{l}\mathrm{p} \\
\text { value }\end{array}$ & $\beta^{a}$ & $\begin{array}{l}\mathrm{p} \\
\text { value }\end{array}$ & $\beta^{a}$ & $\begin{array}{l}\mathrm{p} \\
\text { value }\end{array}$ & $\beta^{\mathrm{a}}$ & $\begin{array}{l}\mathrm{p} \\
\text { value }\end{array}$ & $\beta^{\mathrm{a}}$ & $\begin{array}{l}\mathrm{p} \\
\text { value }\end{array}$ \\
\hline \multirow{6}{*}{$\begin{array}{l}\text { Adjusted } \\
\text { variables }\end{array}$} & Female & -0.051 & 0.248 & -0.053 & 0.244 & -0.055 & 0.214 & 0.037 & 0.420 & 0.032 & 0.486 & 0.037 & 0.415 \\
\hline & $\begin{array}{l}\text { Equivalent } \\
\text { income }\end{array}$ & 0.086 & 0.050 & 0.108 & 0.016 & 0.104 & 0.020 & 0.065 & 0.146 & 0.058 & 0.192 & 0.064 & 0.151 \\
\hline & $\begin{array}{l}\text { Having } \\
\text { present } \\
\text { illness }\end{array}$ & -0.174 & 0.000 & -0.168 & 0.000 & -0.171 & 0.000 & -0.112 & 0.011 & -0.112 & 0.010 & -0.111 & 0.011 \\
\hline & $\begin{array}{l}\text { Need long- } \\
\text { term care }\end{array}$ & -0.003 & 0.945 & 0.002 & 0.966 & 0.008 & 0.854 & -0.128 & 0.004 & -0.127 & 0.004 & -0.128 & 0.003 \\
\hline & $\begin{array}{l}\text { Not living } \\
\text { alone }\end{array}$ & -0.070 & 0.131 & -0.017 & 0.714 & -0.005 & 0.917 & -0.020 & 0.663 & -0.013 & 0.777 & -0.019 & 0.685 \\
\hline & $\begin{array}{l}\text { Having } \\
\text { child/children }\end{array}$ & 0.041 & 0.364 & 0.056 & 0.219 & 0.049 & 0.277 & 0.071 & 0.116 & 0.069 & 0.117 & 0.071 & 0.111 \\
\hline \multirow[t]{6}{*}{ Objects } & $\begin{array}{l}\text { Family or } \\
\text { relatives }\end{array}$ & 0.202 & 0.000 & & & & & 0.001 & 0.990 & & & & \\
\hline & $\begin{array}{l}\text { Individual } \\
\text { friends }\end{array}$ & & & 0.069 & 0.125 & & & & & 0.053 & 0.227 & & \\
\hline & $\begin{array}{l}\text { Community } \\
\text { dwellers }\end{array}$ & & & & & 0.147 & 0.001 & & & & & 0.007 & 0.867 \\
\hline & $\mathrm{R} 2^{\mathrm{b}}$ & 0.068 & & 0.035 & & 0.052 & & 0.029 & & 0.032 & & 0.029 & \\
\hline & $\mathrm{N}$ & 494 & & 494 & & 494 & & 515 & & 515 & & 515 & \\
\hline & $\mathrm{F}$ & 6.138 & & 3.577 & & 4.861 & & 3.216 & & 3.434 & & 3.221 & \\
\hline
\end{tabular}


Table 6. Multiple liner regression analysis modeling objects of provided instrumental social support

\begin{tabular}{|c|c|c|c|c|c|c|c|c|c|c|c|c|c|}
\hline & & \multicolumn{6}{|c|}{ Pre-old $(n=659)$} & \multicolumn{6}{|c|}{ Old ( $n=888)$} \\
\hline & & \multicolumn{2}{|c|}{ Model 1} & \multicolumn{2}{|c|}{ Model 2} & \multicolumn{2}{|c|}{ Model 3} & \multicolumn{2}{|c|}{ Model 4} & \multicolumn{2}{|c|}{ Model 5} & \multicolumn{2}{|c|}{ Model 6} \\
\hline & & $\beta^{\mathrm{a}}$ & $\begin{array}{l}\mathrm{p} \\
\text { value }\end{array}$ & $\beta^{\mathrm{a}}$ & $\begin{array}{l}\mathrm{p} \\
\text { value }\end{array}$ & $\beta^{\mathrm{a}}$ & $\begin{array}{l}\mathrm{p} \\
\text { value }\end{array}$ & $\beta^{\mathrm{a}}$ & $\begin{array}{l}\mathrm{p} \\
\text { value }\end{array}$ & $\beta^{\mathrm{a}}$ & $\begin{array}{l}\mathrm{p} \\
\text { value }\end{array}$ & $\beta^{\mathrm{a}}$ & $\begin{array}{l}\mathrm{p} \\
\text { value }\end{array}$ \\
\hline \multirow{6}{*}{$\begin{array}{l}\text { Adjusted } \\
\text { variables }\end{array}$} & Female & -0.044 & 0.318 & -0.071 & 0.120 & -0.065 & 0.156 & 0.010 & 0.838 & 0.007 & 0.888 & 0.012 & 0.793 \\
\hline & $\begin{array}{l}\text { Equivalent } \\
\text { income }\end{array}$ & 0.084 & 0.062 & 0.110 & 0.013 & 0.105 & 0.019 & 0.078 & 0.092 & 0.075 & 0.102 & 0.081 & 0.078 \\
\hline & $\begin{array}{l}\text { Having } \\
\text { present } \\
\text { illness }\end{array}$ & -0.169 & 0.000 & -0.165 & 0.000 & -0.162 & 0.000 & -0.121 & 0.008 & -0.121 & 0.008 & -0.120 & 0.008 \\
\hline & $\begin{array}{l}\text { Need long- } \\
\text { term care }\end{array}$ & -0.026 & 0.563 & -0.024 & 0.586 & -0.027 & 0.552 & -0.115 & 0.013 & -0.107 & 0.021 & -0.113 & 0.014 \\
\hline & $\begin{array}{l}\text { Not living } \\
\text { alone }\end{array}$ & -0.037 & 0.422 & -0.005 & 0.907 & -0.018 & 0.694 & -0.007 & 0.886 & -0.014 & 0.769 & -0.018 & 0.697 \\
\hline & $\begin{array}{l}\text { Having } \\
\text { child/children }\end{array}$ & 0.038 & 0.413 & 0.048 & 0.292 & 0.045 & 0.328 & 0.045 & 0.325 & 0.046 & 0.319 & 0.046 & 0.316 \\
\hline \multirow[t]{6}{*}{ Objects } & $\begin{array}{l}\text { Family or } \\
\text { relatives }\end{array}$ & 0.152 & 0.001 & & & & & -0.031 & 0.527 & & & & \\
\hline & $\begin{array}{l}\text { Individual } \\
\text { friends }\end{array}$ & & & 0.145 & 0.001 & & & & & 0.035 & 0.443 & & \\
\hline & $\begin{array}{l}\text { Community } \\
\text { dwellers }\end{array}$ & & & & & 0.115 & 0.011 & & & & & -0.035 & 0.447 \\
\hline & $\mathrm{R} 2^{\mathrm{b}}$ & 0.053 & & 0.052 & & 0.044 & & 0.025 & & 0.025 & & 0.025 & \\
\hline & $\mathrm{N}$ & 489 & & 489 & & 489 & & 479 & & 479 & & 479 & \\
\hline & $\mathrm{F}$ & 4.941 & & 4.798 & & 4.232 & & 2.762 & & 2.79 & & 2.788 & \\
\hline
\end{tabular}

\section{Discussion}

In this study, we investigated whether the association between social support and mental health varied in different aging stages in relation to objects by integrating sub-dimensions of support such as functions and roles. Our results demonstrated different patterns within three categories of objects; either ESS or ISS from or to community neighbors showed a positive relationship with mental health whereas supportive interaction with kin or friends, as the main objects in a supportive social network among the older people, may lose its positive relation with mental health when it comes to specific subscales of social support (kin: provided ESS; friends: provided ESS or received ISS). Association between social support and mental health varied by the two age groups suggesting social support potentially related to the mental health status of pre-old age rather than the old age respondents. Our analysis showed a different association between specific objects and mental health, which was a novelty of our investigation for filling the blank of existing literature, considering available present publications barely discussed the social supports from the view point of those objects. We will discuss our findings regarding the three object categories respectively as follows.

Firstly; Models 1 in Table 3, Table 5 and Table 6 illustrated beneficial results from social supportive interaction with family and relatives, especially in terms of instrumental social support. Previous reports have shown that perceived support from family has a positive relationship [22], especially one's spouse for married people and children for people living without a spouse [21, 23]. Secondly; our analyses demonstrated that a supportive connection with friends had a positive result on mental health (Models 2 in Table 3 \& Table 6), which was in accordance with previous researches [7, 23]. However, our results show that the relationship only happened when it was received ESS or provided ISS, which suggests a preference for taking less and giving more to friends. This may be influenced by Japanese culture which values being modest and not bothering others; however, further research is needed to clarify the mechanism. 
Thirdly; while previous reports were limited to the level of friends or family, our results show that supportive interaction with community dwellers had a positive relationship with mental health (Models 3 in Table 3, 4, 5, 6). The social supports from or to community dwellers were almost the same in the two age groups (Table 2); however, they were only related with better mental health in the pre-old age adults, especially in terms of ESS. This suggests that promoting community-level interaction from the pre-old stage is important. Cornwell [38] reported weak ties, like contacts with acquaintances or neighbors help with keeping independence for the older people and Stahl et al. [39] reported the beneficial effect of neighborhood social quality on depression for elderly people living alone. Given that family or relatives are connected by marriage or blood and individual friends need time to build and maintain, community dwellers as public resources are available to each community dweller. Our result suggests a feasible low-cost public health intervention by promoting community participation.

Although we hadn't got a clear figure on the effects of social support during the real old age stage, Models 4, 5, and 6 in Table 3 to Table 6 demonstrated a negative pattern of being in long-term care only in the old-age group. The result is supported by some previous studies that indicated that low functional ability or disability was connected with a high incidence rate of depression [40, 41]. In addition, the present research verified the interaction of functional ability and social support and showed that providing family or relatives instrumental social support may mediate the negative relationship between low functional ability and mental health (Figure A1; see Additional file 5). This suggests that an intervention focused on disadvantaged older people groups could be introduced, which values provided social support within the family.

Taken together, our results confirmed the earlier finding [42] about an inward turn to close relationship in terms of social support in later life, as expected for the shrinking social networks documented in earlier findings [43-45]. In contrast, our data of association between mental health and social support suggested a potentially beneficial meaning of supportive interaction with community people. Given the shrinking social networks along with the natural aging process implies fewer interactions with not-so-close relationships like community neighbors or acquaintances, the older people are potentially at risk of losing availabilities of community-based mental-health-friendly support as time goes on. The intervention of community-based support is suggested to be discussed in the future.

Our investigation illustrated beneficial results of being a provider or a receiver of ESS or ISS on mental health, which echo previous studies [18, 46]; but the positive associations were only among pre-old respondents. An available paper revealed a similar outcome of a more significant association on well-being in the younger-old group [47]. A dynamic view of the aging process is suggested here; while the reasons about agebased differences on social support and mental health haven't been well documented. Our data depicted a positive association of total received ESS in both pre-old and old groups (Table A1; see Additional file 6), while results in respect of objects varied according to two age phases (Table 3). Subdivision of objects could be an inspiration that contributes to clarifying the age-specific mechanism of social support and mental health in the future.

There were some limitations to our research. First, we used a cross-sectional sample which limited our ability to establish a causal relationship. Second, although quota sampling was conducted to compare different ages and subdivisions of social support, it introduced a weakness concerning representation and generalization of results. Third, few items of social support were designed to ease the burden of filling up the questionnaire, considering the physical and comprehension limitations of the older people. More details on the multiple subdimensions and need to be investigated in future research.

\section{Conclusion}

The association between social support and mental health in later life differed based on the objects of social support regarding subscales of roles, functions. The association also varied by age; a positive relationship was found only in the pre-old group. Physical disability has a marked effect on the old age individual's mental health. Public health policy should consider the potential effects of social interaction with community neighbors.

\section{Abbreviations}

ESS: emotional social support

ISS『instrumental social support

\section{Declarations}

\section{Availability of data and materials}

The datasets used and/or analyzed during the current study are available from the corresponding author on reasonable request. 


\section{Ethics approval and consent to participate}

The study was approved by the ethics committee at the Division of Health Sciences, Osaka University Graduate School of Medicine (approval number 271). All participants provided written informed consent.

\section{Consent for publication}

Not applicable.

\section{Competing interests}

The authors declare that they have no competing interests.

\section{Acknowledgments}

We are grateful to all participants in this study.

\section{Funding}

This study was supported in part by grants-in-aid from the JSPS KAKENHI (MK : 16K12336, 19K11138). The grants were for the data collection, analysis, and interpretation of data; and in writing the manuscript.

\section{Authors Contributions}

YL, MK, KK: concept, designed and developed the overall research plan. MK, KK: data collection. YL: data analysis, and interpretation of data. YL, MK, KK: wrote and had responsibility for the final contents of the manuscript. All the authors read and approved the final manuscript.

\section{References}

1. WHO: Mental Health of Older Adults. 2017. https://www.who.int/news-room/fact-sheets/detail/mental-health-of-older-adults. Accessed 21 May 2020.

2. Berkman LF, Glass T, Brissette I, Seeman TE. From social integration to health: Durkheim in the new millennium. Soc Sci Med. 2000;51:843-57.

3. Chan YK, Lee RPL. Network size, social support and happiness in later life: a comparative study of Beijing and Hong Kong. J Happiness Stud. 2006;7:87-112.

4. Chao SF. Assessing social support and depressive symptoms in older Chinese adults: a longitudinal perspective. Aging Ment Heal. 2011;15:765-74.

5. Mohd TAMT, Yunus RM, Hairi F, Hairi NN, Choo WY. Social support and depression among community dwelling older adults in Asia: A systematic review. BMJ Open. 2019;9.

6. Cobb S. Social support as a moderator of life stress. Psychosom Med. 1976;38:300-14.

7. Wu F, Sheng Y. Differences in social isolation between young and old elderly in urban areas of Beijing, China: a cross-sectional study. Int $\mathrm{J}$ Nurs Sci. 2020;7:49-53.

8. Feng Z, Jones K, Wang WW. An exploratory discrete-time multilevel analysis of the effect of social support on the survival of elderly people in China. Soc Sci Med. 2015;130:181-9..

9. Shye D, Mullooly JP, Freeborn DK, Pope CR. Gender differences in the relationship between social network support and mortality: A longitudinal study of an elderly cohort. Soc Sci Med. 1995;41:935-47.

10. Hatfield JP, Hirsch JK, Lyness JM. Functional impairment, illness burden, and depressive symptoms in older adults: does type of social relationship matter? Int J Geriatr Psychiatry. 2013;28:190-8.

11. Taylor MG, Lynch SM. Trajectories of impairment, social support, and depressive symptoms in later life. Journals Gerontol - Ser B Psychol Sci Soc Sci. 2004;59:238-46. 
12. Graham C, Pozuelo JR. Happiness, stress, and age: how the U-Curve varies across people and places. J Popul Rconomics. 2016;30:22564.

13. Sigelman CK, Rider EA. Life-span human development, 6th ed. Belmont, CA: Wadsworth/Cengage Learning; 2009.

14. Van Tilburg T. Losing and gaining in old age: Changes in personal network size and social support in a four-year longitudinal study. Journals Gerontol - Ser B Psychol Sci Soc Sci. 1998;53:313-23.

15. Huxhold O, Fiori KL, Windsor TD. The dynamic interplay of social network characteristics, subjective well-being, and health: the costs and benefits of socio-emotional selectivity. Psychol Aging. 2013;28:3-16.

16. Morgan DL, Schuster TL, Butler EW. Role reversals in the exchange of social support. Journals Gerontol. 1991;46:278-87.

17. Pruchno RA, Burant CJ, Peters ND. Understanding the well-being of care receivers. Gerontologist. 1997;37:102-9.

18. Lu S, Wu Y, Mao Z, Liang X. Association of formal and informal social support with health-related quality of life among Chinese rural elders. Int J Environ Res Public Health. 2020;17.

19. Seeman TE, Berkman LF. Structural characteristics of social networks and their relationship with social support in the elderly: who provides support. Soc Sci Med. 1988;26:737-49.

20. Liao CC, Yeh CJ, Lee SH, Liao WC, Liao MY, Lee MC. Providing instrumental social support is more beneficial to reduce mortality risk among the elderly with low educational level in Taiwan: a 12-year follow-up national longitudinal study. J Nutr Heal Aging. 2014;19:44753.

21. Okabayashi H, Liang J, Krause N, Akiyama H, Sugisawa H. Mental health among older adults in Japan: do sources of social support and negative interaction make a difference? Soc Sci Med. 2004;59:2259-70.

22. Bélanger E, Ahmed T, Vafaei A, Curcio CL, Phillips SP, Zunzunegui MV. Sources of social support associated with health and quality of life: a cross-sectional study among Canadian and Latin American older adults. BMJ Open. 2016;6.

23. Li H, Ji Y, Chen T. The roles of different sources of social support on emotional well-being among Chinese elderly. PLoS One. 2014;9:1-8.

24. Liu X, Liang J, Gu S. Flows of social support and health status among older persons in China. Soc Sci Med. 1995;41:1175-84.

25. Mizuno Y, Hikichi H, Noguchi M, Kawachi I, Takao S. Reciprocity of social support is associated with psychological distress and suicidal ideation in older Japanese people: a population-based study. Soc Sci Med. 2019;230 April:131-7.

26. Hirakata. Population of Hirakata City in Oct.2013. https://www.city.hirakata.osaka.jp/cmsfiles/contents/0000001/1084/41087.pdf. Accessed 21 May 2020.

27. Hall ET. The Hidden Dimension. New York: Double Day;1990.

28. House JS, Umberson D, Landis KR. Structures And Processes Of Social Support. Annu Rev Sociol. 1988;14:293-318.

29. Semmer NK, Elfering A, Jacobshagen N, Perrot T, Beehr TA, Boos N. The Emotional Meaning of Instrumental Social Support. Int J Stress Manag. 2008;15:235-51.

30. IHope. MOS 36-Item Short-Form Health Survey. 2019. https://www.sf-36.jp/qol/sf36.html. Accessed 21 May 2020.

31. Fukuhara S, Ware JE, Kosinski M, Wada S, Gandek B. Psychometric and clinical tests of validity of the Japanese SF-36 Health Survey. J Clin Epidemiol. 1998;51:1045-53.

32. Fukuhara S, Bito S, Green J, Hsiao A, Kurokawa K. Translation, adaptation, and validation of the SF-36 Health Survey for use in Japan. J Clin Epidemiol. 1998;51:1037-44.

33. Suzukamo Y, Fukuhara S, Green J, Kosinski M, Gandek B, Ware JE. Validation testing of a three-component model of Short Form-36 scores. J Clin Epidemiol. 2011;64:301-8.

34. Fukuhara S, Suzukamo Y. Manual of SF-36v2 Japanese version: Institute for Health Outcomes \& Process Evaluation research. Kyoto; 2004.

35. Ouchi Y, Rakugi H, Arai H, Akishita M, Ito H, Toba K, et al. Redefining the elderly as aged 75 years and older: Proposal from the Joint Committee of Japan Gerontological Society and the Japan Geriatrics Society. Geriatr Gerontol Int. 2017;17:1045-7.

36. Kabayama M, Watanabe C, Ryuno H, Kamide K. Positive and negative associations of individual social capital factors with health among community-dwelling older people. Geriatr Gerontol Int. 2017;17:2427-34.

37. MHLW. Comprehensive Survey of Living Conditions:the year of 2013. 2014. https://www.mhlw.go.jp/toukei/saikin/hw/k-tyosa/ktyosa13/dl/16.pdf. Accessed 21 May 2020.

38. Cornwell B. Independence through social networks: Bridging potential among older women and men. Journals Gerontol - Ser B Psychol Sci Soc Sci. 2011;66 B:782-94.

39. Stahl ST, Beach SR, Musa D, Schulz R, Stahl ST, Beach SR, et al. Living alone and depression : the modifying role of the perceived neighborhood environment. Aging Ment Health. 2016;21:1065-71.

Page $12 / 13$ 
40. Li X, Wang W, Gao Q, Wu L, Luo Y, Tang Z, et al. The trajectories and correlation between physical limitation and depression in elderly residents of Beijing, 1992-2009. PLoS One. 2012;7:1-7.

41. Kwon M, Kim SA, Seo K. Systematic review on the relationship between depressive symptoms and Activities of Daily Living in cognitively intact older adults. Korean J Adult Nurs. 2019;31:1-13.

42. Field D, Minkler M. Continuity and change in social support between young-old and old-old or very-old age. Journals Gerontol. 1988;43:100-6.

43. Broese Van Groenou M, Hoogendijk EO, Van Tilburg TG. Continued and new personal relationships in later life: Differential effects of health. J Aging Health. 2013;25:274-95.

44. Fiori KL, Antonucci TC, Akiyama H. Profiles of social relations among older adults: A cross-cultural approach. Ageing Soc. 2008;28:20331.

45. Wrzus C, Hänel M, Wagner J, Neyer FJ. Social network changes and life events across the life span: A meta-analysis. Psychol Bull. 2013;139:53-80.

46. Thomas PA. Is it better to give or to receive? Social support and the well-being of older adults. J Gerontol B Psychol Sci Soc Sci. 2010;65 B:351-7.

47. Litwin H, Stoeckel KJ. Social networks and subjective wellbeing among older Europeans: Does age make a difference? Ageing Soc. 2013;33:1263-81.

\section{Supplementary Files}

This is a list of supplementary files associated with this preprint. Click to download.

- Additionalfile4.xls

- Additionalfile1.xls

- Additionalfile3.xls

- Additionalfile2.xls

- Additionalfile6.xls

- Additionalfile5.doc 